\title{
Recomendações para o Manejo de Dispositivos Cardíacos Eletrônicos Implantáveis Post Mortem
}

Recommendations for the Post-Mortem Management of Cardiac Implantable Electronic Devices

Júlio César de Oliveira, ${ }^{1,2}$ Alexsandro Alves Fagundes, ${ }^{3}$ Ricardo Alkmim-Teixeira, ${ }^{4,5}$ (osé Mário Baggio Junior, ${ }^{6}$ Luciana Armaganijan, ${ }^{7}$ Andre d'Avila, ${ }^{8}$ (1) Eduardo B. Saad, ${ }^{9}$ Veridiana Silva de Andrade, ${ }^{10}$ (ㄴ) Luis Gustavo Belo de Moraes, ${ }^{11}$ Ricardo Kuniyoshi, ${ }^{12}$ André Gustavo da Silva Rezende, ${ }^{13,14}{ }^{\circledR}$ Mauricio Pimentel, ${ }^{15}$ Thiago da Rocha Rodrigues, ${ }^{16}$ Helio Lima de Brito Junior, ${ }^{17}$ Elenir Nadalin, ${ }^{18,19,20}$ Cristiano Faria Pisani, ${ }^{21}{ }^{\circledR}$ Elerson Arfelli, ${ }^{22}$ Fatima Dumas Cintra, ${ }^{10}$ Carlos Antonio Abunader Kalil, ${ }^{23,24}$ Sissy Lara de Melo, ${ }^{21}$ Priscila Moreno Sperling Cannavan ${ }^{25}$ Universidade Federal de Mato Grosso, ${ }^{1}$ Cuiabá, MS - Brasil

Hospital Geral Filantrópico Universitário de Cuiabá, ${ }^{2}$ Cuiabá, MS - Brasil

Universidade do Estado da Bahia, ${ }^{3}$ Salvador, BA - Brasil

Hospital Renascentista, ${ }^{4}$ Pouso Alegre, MG - Brasil

Universidade do Vale do Sapucaí, ${ }^{5}$ Pouso Alegre, MG - Brasil

Instituto de Cardiologia do Distrito Federal, ${ }^{6}$ Brasília, DF - Brasil

Instituto Dante Pazzanese de Cardiologia, ${ }^{7}$ São Paulo, SP - Brasil

Hospital SOS Cardio, ${ }^{8}$ Florianópolis, SC - Brasil

Hospital Pró-Cardíaco, ${ }^{9}$ Rio de Janeiro, RJ - Brasil

Universidade Federal de São Paulo, ${ }^{10}$ São Paulo, SP - Brasil

Universidade Federal do Rio de Janeiro Hospital Universitário Clementino Fraga Filho, ${ }^{11}$ Rio de Janeiro, RJ - Brasil

Centrocor - Cardiologia, ${ }^{12}$ Vitória, ES - Brasil

Hospital das Clinicas da Universidade Federal de Pernambuco - Medicina Clínica, ${ }^{13}$ Recife, PE - Brasil

Pronto Socorro Cardiológico de Pernambuco (Procape) - Universidade de Pernambuco, ${ }^{14}$ Recife, PE - Brasil

Hospital de Clínicas de Porto Alegre, ${ }^{15}$ Porto Alegre, RS - Brasil

Hospital Felício Rocho, ${ }^{16}$ Belo Horizonte, MG - Brasil

Universidade Federal de Juiz de Fora, ${ }^{17}$ Juiz de Fora, MG - Brasil

Hospital Cardiológico Costantini Ltda, ${ }^{18}$ Curitiba, PR - Brasil

Hospital Marcelino Champagnat, ${ }^{19}$ Curitiba, $P R$ - Brasil

Irmandade da Santa Casa de Misericórdia de Curitiba, ${ }^{20}$ Curitiba, PR - Brasil

Universidade de São Paulo Faculdade de Medicina Hospital das Clínicas Instituto do Coração, ${ }^{21}$ São Paulo, SP - Brasil

Universidade de São Paulo Hospital das Clínicas da Faculdade de Medicina de Ribeirão Preto, ${ }^{22}$ Ribeirão Preto, SP - Brasil

Complexo Hospitalar Santa Casa de Porto Alegre, ${ }^{23}$ Porto Alegre, RS - Brasil

Pontifícia Universidade Católica do Rio Grande do Sul, ${ }^{24}$ Porto Alegre, RS - Brasil

Universidade Estadual de Campinas - Faculdade de Enfermagem, ${ }^{25}$ Campinas, SP - Brasil

\section{Resumo}

O manejo de dispositivos cardíacos eletrônicos implantáveis de pacientes que evoluem a óbito tem sido motivo de controvérsia. Em nosso meio, não há recomendações uniformes, estando baseadas exclusivamente em protocolos institucionais e em costumes regionais. Quando o cadáver é submetido para cremação, além de outros cuidados, recomenda-se a retirada do dispositivo devido ao risco de explosão e dano do equipamento crematório. Principalmente no contexto da pandemia causada

\section{Palavras-chave}

Dispositivos Cardíacos Eletrônicos Implantáveis/ complicações; Ética Baseada em Princípios; Autópsia/ métodos.

Correspondência: Ricardo Alkmim Teixeira •

Sociedade Brasileira de Arritmias Cardíacas - Alameda dos Maracatins, 1435 CEP 04089-015, Conj. 301/306, São Paulo, SP - Brasil

E-mail: ricardo.alkmim@gmail.com

Artigo recebido em 09/05/2020, revisado em 24/06/2020, aceito em $24 / 06 / 2020$

DOI: https://doi.org/10.36660/abc.20200447 pelo SARS-Cov-2, a orientação e organização de unidades hospitalares e serviços funerários é imprescindível para minimizar o fluxo de pessoas em contato com fluidos corporais de indivíduos falecidos por COVID-19. Nesse sentido, a Sociedade Brasileira de Arritmias Cardíacas elaborou este documento com orientações práticas, tendo como base publicações internacionais e recomendação emitida pelo Conselho Federal de Medicina do Brasil.

O número de implantes de dispositivos cardíacos eletrônicos implantáveis (DCEI) (marcapassos, ressincronizadores cardíacos, cardioversores-desfibriladores implantáveis e monitores de eventos) tem aumentado consideravelmente nas últimas décadas devido ao grande avanço em seus recursos diagnósticos e terapêuticos, além do evidente aumento da longevidade dos pacientes (pacientes idosos podem ter maior longevidade graças aos DCEI). Dessa forma, o aumento de situações clínicas em que o uso desses dispositivos se aplica é acompanhado por aumento de complicações e outros problemas a eles relacionados.

Uma grande preocupação em relação aos $\mathrm{DCEl}$, principalmente na população de idosos, em decorrência das taxas de mortalidade relacionadas à infecção pelo SARS-Cov-2 (COVID-19), é a conduta após o óbito: 
- O DCEI deve ser desligado?

- O DCEI deve ser removido para o sepultamento?

- O cadáver vai ser cremado. Quais cuidados devemos tomar?

- No caso de remoção do DCEI, quem deve realizar o procedimento?

Respondendo a esses questionamentos, a Sociedade Brasileira de Arritmias Cardíacas (SOBRAC) elaborou algumas recomendações baseadas em evidências internacionais., que julgamos ser imprescindíveis para o esclarecimento de médicos, hospitais e serviços funerários que lidam diariamente com essa situação:

1. Em pacientes que faleceram de morte súbita, os DCEI devem ser submetidos a avaliação eletrônica quando o médico responsável pelo paciente considerar necessário o esclarecimento da causa da morte, uma vez que os dispositivos dispõem de recursos que possibilitam a gravação e o armazenamento do ritmo cardíaco nesses casos.

2. Em portadores de DCEl que serão sepultados, não se faz necessária a reprogramação ou remoção do aparelho.

3. Portadores de DCEl que serão submetidos à cremação devem, obrigatoriamente, ter seu aparelho removido devido ao risco de explosão causada pelo superaquecimento (cerca de $1.300^{\circ} \mathrm{C}$ por 90 minutos) e danos ao equipamento crematório.

4. Os cardioversores-desfibriladores implantáveis (CDI) de cadáveres que serão cremados ou que serão submetidos a autópsia devem ter as terapias desabilitadas por telemetria, a fim de prevenir o disparo de choques durante a manipulação do CDI pelo profissional que realiza o trabalho. A reprogramação por telemetria deve ser realizada por médico com área de atuação em estimulação cardíaca eletrônica implantável ou por técnico devidamente qualificado. Para a autópsia, eventualmente se pode considerar, como alternativa em casos excepcionais, a colocação do imã sobre o gerador do CDI em vez da reprogramação por telemetria. Nesse caso, o imã deve ser mantido sobre o gerador mesmo após a sua retirada, uma vez que o risco de choque persiste até que as terapias sejam desabilitadas com programação eletrônica.

5. De acordo com ofício expedido pelo Conselho Federal de Medicina (anexo) em 05 de maio de 2020, atendendo à solicitação da SOBRAC, sob o número 2.628/2020-DEPCO, a retirada dos DCEI post mortem deve ser realizada "preferencialmente por médico e devidamente registrado no prontuário do paciente, resguardando a segurança no momento da pandemia COVID-19, por isso, a importância do envolvimento da diretoria técnica para criar o melhor protocolo para a instituição". A SOBRAC alerta que o profissional não médico (seja do serviço funerário ou da própria unidade hospitalar) que eventualmente seja designado para essa tarefa deve ser devidamente treinado, ainda que tal procedimento exija técnica simples e não coloque em risco a vida do profissional que executa o procedimento.

O entendimento dos aspectos relacionados ao manuseio de DCEl após o óbito, em momento de pandemia pela COVID-19, é fundamental para reduzir o risco de disseminação da doença.

Abaixo, descrevemos a técnica de remoção do DCEl:

- paramentação apropriada para o procedimento, a fim de evitar a contaminação do profissional através de fluidos corporais;

- utilizar lâmina de bisturi para incisar a pele profundamente em cima do gerador de pulsos;

- divulsionar o tecido profundo com os dedos ou tesoura;

- o gerador deve ser puxado para fora, cortando-se os eletrodos com tesoura;

- fechar a incisão com gaze e esparadrapo, não sendo necessária sutura;

- descartar o dispositivo de forma segura, segundo recomendações técnicas e protocolos institucionais.

\section{Contribuição dos autores}

Concepção e desenho da pesquisa e Obtenção de dados: Oliveira JC, Fagundes AA, Teixeira RA; Análise e interpretação dos dados: Oliveira JC, Fagundes AA, Teixeira RA; Baggio Junior JM, Armaganijan L, d'Avila A, Saad EB, Andrade VS, Moraes LGB, Kuniyoshi R, Rezende AGS, Pimentel M, Rodrigues TR, Brito Junior HL, Pisani CF, Arfelli E, Cintra FD, Kalil CAA, Melo SL, Cannavan PMS; Análise estatística: Nadalin E; Redação do manuscrito: Oliveira JC, Fagundes AA, Teixeira RA, Baggio Junior JM, Armaganijan L, d'Avila A, Saad EB, Andrade VS, Moraes LGB, Kuniyoshi R; Revisão crítica do manuscrito quanto ao conteúdo intelectual importante: Oliveira JC, Fagundes AA, Teixeira RA, Baggio Junior JM, Armaganijan L, d'Avila A, Saad EB, Andrade VS, Moraes LGB, Kuniyoshi R, Rezende AGS, Pimentel M, Rodrigues TR, Brito Junior HL, Nadalin E, Pisani CF, Arfelli E, Cintra FD, Kalil CAA, Melo SL, Cannavan PMS.

\section{Potencial conflito de interesses}

Declaro não haver conflito de interesses pertinentes.

\section{Fontes de financiamento}

O presente estudo não teve fontes de financiamento externas.

\section{Vinculação acadêmica}

Não há vinculação deste estudo a programas de pósgraduação.

\section{Referências}

1. Gale CP, Mulley GP. Pacemaker explosions in crematoria: problems and possible solutions. J R Soc Med. 2002;95(7):353-5.

2. Pitcher D, Soar J, Hogg K, Linker N, Chapman S, Batle J, et al. Cardiovascular implanted electronic devices in people towards the end of life, during cardiopulmonary resuscitation and after death: guidance from the Resuscitation Council (UK), British Cardiovascular Society and National Council for Palliative Care. Heart. 2016;102 (Suppl 7):A1-A17. 